\title{
Surveillance of HIV Type 1 Recent Infection and Molecular Epidemiology Among Different Risk Behaviors Between 2007 and 2009 After the HIV Type 1 CRF07_BC Outbreak in Taiwan
}

\author{
Cheng-Feng Kao,, ${ }^{1,}$ Sui-Yuan Chang, ${ }^{2,}{ }^{\star}$ Kan-Tai Hsia, ${ }^{3,{ }^{*}}$ Feng-Yee Chang, ${ }^{4}$ Chin-Hui Yang, ${ }^{4}$ \\ Hui-Rong Liu, ${ }^{4}$ Tzu-Yu Fu, and Jyh-Yuan Yang ${ }^{5}$
}

\begin{abstract}
The objective of this study was to analyze recent infections and the molecular epidemiology of human immunodeficiency virus type 1 (HIV-1) among different risk groups since the outbreak of circulating recombinant form CRF07_BC among intravenous drug users (IDUs) in 2004 in Taiwan. Phylogenetic analysis was performed using the env and pol fragment sequences amplified from these specimens. The BED IgG capture incidence EIA (BED-CEIA assay) was used to determine recent infections. Among the $683 \mathrm{HIV}$-1-positive individuals enrolled between 2007 and 2009, 394 (57.7\%) were subtype B, 260 (38.1\%) were CRF07_BC, 26 (3.8\%) were CRF01_AE, two $(0.3 \%)$ were CRF08_BC, and one $(0.1 \%)$ was CRF06_cpx. While the percentage of CRF07_BC decreased (58.5$17.9 \%, p<0.001)$ from 2007 to 2009 , the percentage of subtype B increased ( $37.6 \%$ to $74.9 \%, p<0.001)$. A concordant decrease in the proportion of recent infections to new infections among IDUs $(63.6 \%$ to $9.8 \%, p<0.001)$, accompanied with an increase of the proportion of recent infections in MSM (men having sex with men) (22.4$67.1 \%, p=0.77)$ and heterosexual groups (13.1-23.2\%, $p=0.852)$, was observed. The decrease in CRF07_BC infections and the reduction in the proportion of recent infections among IDUs reflected the success of harm reduction strategies initiated by the government in 2005 .
\end{abstract}

\section{Introduction}

$\mathbf{H}$ IV IS THE CAUSATIVE AGENT of acquired immunodeficiency syndrome (AIDS) and has caused a worldwide epidemic. Based on the phylogenetic analysis of HIV env and/ or gag sequences, HIV can be divided into three groups, M (major), O (outlier), and N (new). More than 99\% of the HIV-1 strains isolated from HIV-infected individuals belong to group $\mathrm{M}$, which can be divided into nine subtypes (A-D, F-H, J, and $\mathrm{K})$ and 16 major circulating recombinant forms (CRFs). ${ }^{1,2} \mathrm{Ge}-$ netic subtyping has been used to track the prevalence of different subtypes in the world and the changes in subtype distribution along with time. In Asia, the HIV-1 epidemic is mainly caused by subtype B', a variant subtype B virus isolated in Thailand, China, Malaysia, and Japan, ${ }^{1}$ subtype C in India, and CRF01_AE in Southeast Asia.
In Taiwan, the first indigenous AIDS case was reported in $1985 .^{2}$ By the end of 2009, a cumulative total of 18,378 HIV-1infected individuals was reported to the Taiwan Centers for Disease Control. ${ }^{3}$ The distribution of HIV-1 subtype viruses from HIV-1-infected persons reported between 1988 and 2000 indicated that subtype B $(69.4 \%)$ was the most prevalent, followed by CRF01_AE (27.3\%) and subtype C $(1.3 \%) ., 4$ Nevertheless, an outbreak of HIV-1 CRF07_BC infections among intravenous drug users (IDUs) in Taiwan in $2004^{6-8}$ dramatically increased the percentage of individuals infected with HIV-1 CRF07_BC (53.4\%), which then became the predominant HIV-1 subtype in Taiwan. ${ }^{9}$

To control the spread of HIV among IDUs, the government implemented a "Harm Reduction Programme" in August 2005, which is composed of three parts as described below. (1) Information, education, and communication. Expanding HIV

\footnotetext{
${ }^{1}$ Institute of Oral Biology, School of Dentistry, National Yang-Ming University, and Research and Diagnostic Center, Centers for Disease Control, Taipei, Taiwan.

${ }^{2}$ Department of Clinical Laboratory Sciences and Medical Biotechnology, National Taiwan University, Taipei, Taiwan.

${ }^{3}$ Institute of Oral Biology, School of Dentistry, National Yang-Ming University, Taipei, Taiwan.

${ }^{4}$ Centers for Disease Control, Taipei, Taiwan.

${ }^{5}$ Research and Diagnostic Center, Centers for Disease Control, Taipei, Taiwan.

*These authors contributed equally to this work.
} 
testing and monitoring of drug users were adapted to detect new cases and provide timely health education information and communication to correct dangerous behavior. (2) A needle-syringe program (NSP). By November 2010, 930 sites provided IDUs with clean syringe paraphernalia, dilution buffer, educational materials, guidance, and counseling to decrease HIV transmission by the use of HIV-contaminated needles and syringes. (3) Methadone substitution treatment. The major thrust of methadone substitution treatment is providing long-term oral methadone treatment to IDUs as a substitute for comparatively risky intravenous drug use. ${ }^{10}$ By November 2010, 100 hospitals and clinics offered substitution treatment services in Taiwan.

The aim of this study was to monitor the distribution of HIV-1 subtypes and HIV-1 recent infections among different risk groups between 2007 and 2009 in Taiwan after the HIV-1 CRF07_BC outbreak in 2004, and to evaluate the effectiveness of the harm reduction program initiated by the government in 2005.

\section{Materials and Methods}

\section{Specimen collection}

Stratified sampling weighted for the proportion of HIV-1seropositive patients among different risk groups was performed, and the newly diagnosed patients were selected from different risk groups based on the specified proportion in each year (2007-2009). A total of 683 HIV-seropositive individuals who were reported to the Taiwan Centers for Disease Control between 2007 and 2009 were enrolled for the study. All blood samples were confirmed for HIV infection by Western blot assay (WB) (New LAV Blot I; Bio-Rad, Marnes la Coquette, France) or polymerase chain reaction (PCR) assay (RealTime HIV-1; Abbott, Des Plaines, IL). This study was reviewed and approved by the ethics committee of the Taiwan Centers for Disease Control (DOH97-CDC-2011, DOH98-CDC-2016).

\section{HIV-1 seroconversion}

An IgG capture enzyme immunoassay was used to measure the proportion of HIV-1-specific IgG in blood samples among the total IgG for the purpose of identifying infections that were acquired recently. The BED-CEIA (capture enzyme immunoassay; Calypte, MD) is a commercial product, using a trimeric branched peptide. Each branch is composed of a synthetic oligopeptide derived from the immunodominant region of the gp41 glycoprotein of HIV-1 subtypes B, CRF01_AE, and D. ${ }^{11}$ It was performed according to the manufacturer's instructions by technicians trained in the use of the assay. Briefly, test specimens were initially run singly. If the normalized OD (ODn) was $>1.2$, the specimen was classified as being from a long-term seroconverter. Specimens with ODn $\leq 1.2$ were tested again in triplicate to confirm the values. In confirmatory testing, specimens with ODn values $\leq 0.8$ were considered a recent seroconversion. ${ }^{12}$ If the ODn of the specimen was $>0.8$, the specimen was considered a longterm seroconversion.

\section{Nested PCR}

HIV-1 viral RNA was extracted from plasma samples with the QIAamp Viral RNA kit (Qiagen, CA) according to the manufacturer's instructions. Nested PCR was performed to generate the $\mathrm{C} 2-\mathrm{V} 3$ fragment of the env gene (nucleotide number, nt 7368-8704) and the pol fragment, containing the entire protease region and the first 335 codons of the reverse transcriptase (nt 2253-3548). ${ }^{9}$ Briefly, the C2-V3 fragment was amplified using a Superscript III One-Step RT-PCR kit (Invitrogen, Carlsbad, CA). The broadly reactive first primer set was 1-CV-F (5'-ACA GTR CAR TGY ACA CAT GG-3') and 1-CV-R (5'-CAC TTC TCC AAT TGT CCI TCA-3'). Thermal cycling conditions were composed of a reverse transcription reaction of $55^{\circ} \mathrm{C}$ for $30 \mathrm{~min}$ and a PCR reaction as follow: $94^{\circ} \mathrm{C}$ for $2 \mathrm{~min}, 35$ cycles of $94^{\circ} \mathrm{C}$ for $15 \mathrm{~s}, 55^{\circ} \mathrm{C}$ for $30 \mathrm{~s}, 68^{\circ} \mathrm{C}$ for $1.5 \mathrm{~min}$, and $68^{\circ} \mathrm{C}$ for $5 \mathrm{~min}$. The second primer set was $2-\mathrm{CV}-\mathrm{F}$ (5'-CTG TTI AAT GGC AGI CTA GC-3') and 2-CV-R (5'-RAT GGG AGG RGY ATA CAT-3'), and the PCR condition was the same as for the first PCR condition. The pol fragment was generated using the ViroSeq HIV-1 Genotyping System Version 2.8 (Celera Diagnostics, Alameda, CA) according to the manufacturer's recommendations. The RT and PCR amplifications were carried out with appropriate negative controls to detect any possible contamination during the experiments.

\section{Phylogenetic analysis}

The PCR products were subjected to a sequencing reaction using the ABI PRISM 3130 Genetic Analyzer (Applied Biosystems, Foster, CA). Sequence fragments were assembled with the Sequencher 4.1.4 software (Gene Codes, Ann Arbor, $\mathrm{MI})$ and aligned with Clustal W. ${ }^{13}$ Phylogenetic tree analyses of the C2-V3 and pol fragment sequences were performed using the neighbor-joining method based on a maximum composite likelihood model with 1000 bootstrap replicates, using MEGA version 4.0. ${ }^{14}$ DNAML from PHYLIP version 3.6.8 was used to construct a maximum-likelihood tree to verify the topology of taxas shown in the neighbor-joining tree. ${ }^{15}$

\section{Nucleotide sequence accession numbers}

The GenBank accession numbers of sequences used in this study are HQ657357-HQ658039, AB220944, AB253421, AB253429, AB286851, AF004885, AF005494, AF005496, AF061641, AF064699, AF075703, AF077336, AF082394-95, AF084936, AF103818, AF190127-28, AF286226, AF286229, AF286237-38, AF377956, AJ249235-39, AY008715, AY173951, AY180905.1, AY253311, AY271690, AY331295, AY371157-58, AY423387, AY531116.1, AY535659, AY612637, AY772699, DQ2308411, DQ2308421, DQ373066, DQ676872, EF0782782, EF368370, EF368372, EF614151, K03454-55, L39106, U46016, U54771, U71182.1, U88824, U88826, and GU299789-90.

\section{Statistical analysis}

Categorical variables were compared using the $\chi^{2}$ test whereas noncategorical variables were compared using the Student's $t$ test. All tests were two-tailed and a $p$ value $<0.05$ was considered significant.

\section{Results}

\section{The trend of HIV-1 infection among IDUs in Taiwan}

By the end of 2009, a cumulative total of 18,378 HIV-1infected individuals was reported to the Taiwan Centers for Disease Control. Among these, 6330 HIV-1-infected individ- 


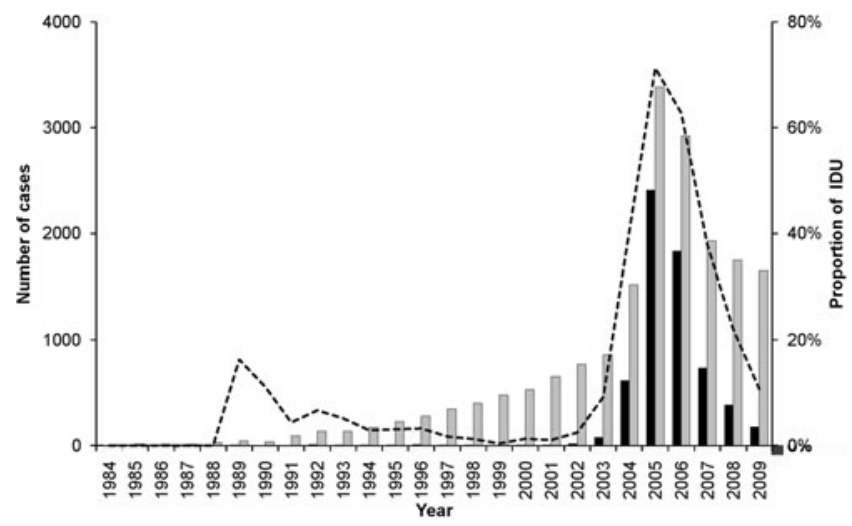

FIG. 1. The trend of HIV-1 infection in IDUs in Taiwan. The dotted line indicates the proportion of IDUs; the gray bar indicates the total number of HIV-1 infections; the black bar indicates the number of IDUs infected with HIV-1.

uals were IDUs. As shown in Fig. 1, the number and percentage of IDUs has increased rapidly since 2002, and reached a peak in 2005. The proportion of IDUs infected with HIV-1 increased gradually from $2.5 \%(19 / 768)$ in 2002 to $9.2 \%$ (79/ $860)$ in 2003 , but jumped to $40.5 \%(616 / 1521)$ in 2004 and to $71.3 \%(2410 / 3381)$ in 2005 . The proportion slowly declined to $62.7 \%(1833 / 2922)$ in 2006 , and further decreased to $37.9 \%$ (733/1932) in 2007, to $22.0 \%$ (384/1746) in 2008 , and to $10.7 \%$ $(177 / 1648)$ in $2009 .^{16}$

\section{Distribution of HIV-1 subtypes and risk behaviors in HIV-infected individuals between 2007 and 2009}

In this cross-sectional study, 683 HIV-1-positive individuals were reported to the Taiwan Centers for Disease Control between 2007 and 2009 and were processed successfully for sequencing and phylogenetic analysis. Among these, 394 $(57.7 \%)$ were subtype B, 260 (38.1\%) were CRF07_BC, 26
(3.8\%) were CRF01_AE, two (0.3\%) were CRF08_BC, and one was CRF06_cpx. The yearly distribution of HIV-1 subtypes is summarized in Table 1. The percentage of subtype B increased from $37.6 \%$ in 2007 to $74.9 \%$ in $2009(p<0.001)$. However, the percentage of CRF07_BC decreased from 58.5\% in 2007 to $17.9 \%$ in $2009(p<0.001)$. The risk behaviors of these individuals were investigated to illustrate the entire picture of the distribution of the risk factors among these HIV-1-positive individuals. As shown in Table 1, the predominant risk behaviors were men having sex with men (MSM) $(44.1 \%)$, IDUs $(33.7 \%)$, and heterosexuals $(21.8 \%)$. Only one case was mother-to-child transmission, and two had unknown risk factors for HIV transmission. While the trends of MSM $(24.5-57.9 \%, p<0.001)$ and heterosexuals $(17.9-26.7 \%$, $p=0.093)$ increased, the trend of IDUs decreased (56.8-15.4\%, $p<0.001)$.

\section{Distribution of HIV-1 subtypes and CRFs among different risk groups}

As shown in Table 2, the prevalence of HIV-1 subtype/CRF in MSM was 295 (98.0\%) subtype B, four (1.3\%) CRF01_AE, and only two (0.7\%) CRF07_BC. In heterosexuals, $90(60.4 \%)$ were subtype B, 37 (24.8\%) were CRF07_BC, 19 (12.8\%) were CRF01_AE, two (1.3\%) were CRF08_BC, and only one (0.7\%) was CRF06_cpx. In IDUs, CRF07_BC was the most prevalent $(95.7 \%)$. In addition, one vertical transmission case was enrolled in this study, which was CRF07_BC.

\section{Recent infections among HIV-1-positive individuals}

Among the 683 HIV-1-positive individuals, 299 individuals $(43.8 \%)$ were identified as recent infections by the BEDCEIA assay. As shown in Table 3, the proportion of recent infections decreased from $46.7 \%$ in 2007 to $42.1 \%$ in 2009 $(p=0.420)$. A drastic increase of MSM in recent infections was observed from $2007(22.4 \%)$ to $2008(68.2 \%)(p<0.001)$, while a great reduction of IDU was simultaneously monitored

Table 1. Baseline Characteristics of HiV-Infected Individuals Enrolled Between 2007 and $2009^{a}$

\begin{tabular}{|c|c|c|c|c|}
\hline & \multirow[b]{2}{*}{ Total } & \multicolumn{3}{|c|}{ Year } \\
\hline & & 2007 & 2008 & 2009 \\
\hline Case number, $N$ & 683 & 229 & 259 & 195 \\
\hline Age, mean $\pm S D$, years & $34.2 \pm 10.2$ & $34.9 \pm 9.8$ & $33.5 \pm 9.9$ & $34.1 \pm 10.9$ \\
\hline Male gender, $N(\%)$ & $642(94.0)$ & $214(93.4)$ & $243(93.8)$ & $185(94.9)$ \\
\hline \multicolumn{5}{|l|}{ Subtype/CRF, N (\%) } \\
\hline $\mathrm{B}, \mathrm{N}(\%)$ & $394(57.7)$ & $86(37.6)$ & $162^{\mathrm{b}}(62.5)$ & $146(74.9)$ \\
\hline CRF01_AE & $26(3.8)$ & $8(3.5)$ & $6(2.3)$ & $12(6.2)$ \\
\hline CRF06_cpx & $1(0.1)$ & $0(0.0)$ & $0(0.0)$ & $1(0.5)$ \\
\hline CRF07_BC & $260(38.1)$ & $134(58.5)$ & $91^{\mathrm{b}}(35.1)$ & $35^{\mathrm{c}}(17.9)$ \\
\hline CRF08_BC & $2(0.3)$ & $1(0.4)$ & $0(0.0)$ & $1(0.5)$ \\
\hline \multicolumn{5}{|l|}{ Risk behavior, $N(\%)$} \\
\hline MSM & $301(44.1)$ & $56(24.5)$ & $132^{\mathrm{b}}(51.0)$ & $113(57.9)$ \\
\hline Heterosexual & $149(21.8)$ & $41(17.9)$ & $56(21.6)$ & $52(26.7)$ \\
\hline IDU & $230(33.7)$ & $130(56.8)$ & $70^{\mathrm{b}}(27.0)$ & $30^{c}(15.4)$ \\
\hline MTCT & $1(0.1)$ & $1(0.4)$ & $0(0.0)$ & $0(0.0)$ \\
\hline Unknown & $2(0.3)$ & $1(0.4)$ & $1(0.4)$ & $0(0.0)$ \\
\hline
\end{tabular}

${ }^{a} \mathrm{CRF}$, circulating recombinant form; MSM, men having sex with men; IDU, intravenous drug user; MTCT, mother-to-child transmission; $\mathrm{SD}$, standard deviation.

${ }^{b}$ Indicates the percentage of HIV subtype/CRF is significantly different $(p<0.05)$ between 2007 and 2008.

'Indicates the percentage of HIV subtype/CRF is significantly different $(p<0.05)$ between 2008 and 2009. 
Table 2. Distribution of HiV-1 Subtype/CRF Among Different Risk Groups in Taiwan Between 2007 and $2009^{a}$

\begin{tabular}{lcccccc}
\hline & \multicolumn{5}{c}{ Subtype/CRF } \\
\cline { 2 - 5 } Risk behavior & $B$ & CRF01_AE & CRF07_BC & CRF08_BC & CRF06_cpx & Total $\mathrm{n}=683(\%)$ \\
\hline MSM $n=301(\%)$ & $295(98.0)$ & $4(1.3)$ & $2(0.7)$ & $0(0.0)$ & $0(0.0)$ & $301(44.1)$ \\
Heterosexual $n=149(\%)$ & $90(60.4)$ & $19(12.8)$ & $37(24.8)$ & $2(1.3)$ & $1(0.7)$ & $149(21.8)$ \\
IDU $n=230(\%)$ & $8(3.5)$ & $2(0.9)$ & $220(95.7)$ & $0(0.0)$ & $0(0.0)$ & $230(33.7)$ \\
MTCT $n=1(\%)$ & $0(0.0)$ & $0(0.0)$ & $1(100.0)$ & $0(0.0)$ & $0(0.0)$ & $1(0.1)$ \\
Unknown $n=2(\%)$ & $1(50.0)$ & $1(50.0)$ & $0(0.0)$ & $0(0.0)$ & $0(0.0)$ & $2(0.3)$ \\
\hline
\end{tabular}

${ }^{a} \mathrm{CRF}$, circulating recombinant form; MSM, men having sex with men; IDU, injection drug user; MTCT, mother-to-child transmission.

(63.6-10.9\%, $p<0.001)$. A similar trend was observed in subtype distribution, with a major increase in subtype B (29.9$85.4 \%, p=0.013)$ and a decrease in CRF07_BC (69.2-12.2\%, $p<0.001)$.

\section{Discussion}

The AIDS Prevention and Control Act from December 1990 serves as the legal basis for the HIV / AIDS control policy in Taiwan. The Act stipulates that clinicians have the responsibility to report HIV/AIDS cases, within $24 \mathrm{~h}$, to local health authorities. For active surveillance, the Taiwan government has been undertaking mandatory screening of blood donors for HIV since 1988, military draftees since 1989, prison inmates since 1990, and foreign laborers since 1991. A screening program for pregnant women was launched in 2005. Although there is no mandatory HIV testing for pregnant women, each of them will receive an HIV / AIDS educational pamphlet and be encouraged by their obstetricians to take a free HIV test (paid by the government) during prenatal care. ${ }^{17}$

Before 2004, the HIV epidemic grew slowly and the major transmission pathway for HIV infection was sexual contacts. However, the number of newly reported HIV-positive individuals has risen sharply since 2004, and IDUs have become the largest contributing risk behavior to the HIV/AIDS epidemic in Taiwan. With the first HIV-infected IDU reported in 1988, subtype B was the predominant subtype among IDUs and the prevalence was kept very low for almost 15 years. ${ }^{18}$
However, an outbreak of HIV-1 CRF07_BC infections among IDUs was reported in 2004. Ninety-eight percent of IDUs were infected with CRF07_BC, which thus surpassed subtype B and became the predominant subtype among HIV-infected individuals in Taiwan. ${ }^{19}$

The HIV incidence in cities with an NSP decreased significantly from 13.9 to 13.3 per 100,000 persons, while an increase of HIV incidence from 11.5 to 15.3 per 100,000 persons was observed in cities without an NSP. ${ }^{10}$ The number and percentage of IDUs have increased rapidly since 2002, and reached a peak in 2005, then decreased after 2006. As shown in Table 1, the percentage of CRF07_BC decreased significantly from $58.5 \%$ in 2007 to $17.9 \%$ in 2009 . Furthermore, the proportion of IDU in individuals who showed evidence of recent infection also decreased significantly $(63.6-9.8 \%, p<0.001)$. These data indicate that the Harm Reduction Program indeed decreased the spread of HIV among IDUs in Taiwan.

The distribution of certain HIV subtypes in different risk groups was noticed (Table 2). As reported previously, 6,20 subtype B and CRF07_BC were mainly circulating among MSM and IDU groups, respectively. No introduction of CRF07_BC into the MSM group was observed. The observation that $24.8 \%$ of heterosexuals were infected with CRF07_BC might be derived from survey bias. Since injection drug use was a criminal offense in Taiwan, some IDUs might be afraid of identifying themselves as IDUs. Such a bias might underestimate the distribution of IDUs and is a major limitation in our study.

Table 3. Characteristics of HiV Patients Identified as Recent Infection Between 2007 and $2009^{a}$

\begin{tabular}{|c|c|c|c|c|c|}
\hline Recent infection case number & $\begin{array}{c}\text { Total } \\
\mathrm{n}=299\end{array}$ & $\begin{array}{c}2007 \\
\mathrm{n}=107\end{array}$ & $\begin{array}{c}2008 \\
\mathrm{n}=110\end{array}$ & $\begin{array}{c}2009 \\
\mathrm{n}=82\end{array}$ & $\mathrm{p}$ value $^{\mathrm{b}}$ \\
\hline \multicolumn{6}{|l|}{ Gender } \\
\hline Male & $288(96.3 \%)$ & $101(94.4 \%)$ & $107(97.3 \%)$ & $80(97.6 \%)$ & 0.420 \\
\hline Female & $11(3.7 \%)$ & $6(5.6 \%)$ & $3(2.7 \%)$ & $2(2.4 \%)$ & 0.224 \\
\hline \multicolumn{6}{|l|}{ Risk behavior } \\
\hline MSM & $154(51.5 \%)$ & $24(22.4 \%)$ & $75(68.2 \%)$ & $55(67.1 \%)$ & 0.770 \\
\hline Heterosexual & $56(18.7 \%)$ & $14(13.1 \%)$ & $23(20.9 \%)$ & $19(23.2 \%)$ & 0.852 \\
\hline IDU & $88(29.4 \%)$ & $68(63.6 \%)$ & $12(10.9 \%)$ & $8(9.8 \%)$ & $<0.001$ \\
\hline Others & $1(0.3 \%)$ & $1(0.9 \%)$ & $0(0.0 \%)$ & $0(0.0 \%)$ & \\
\hline \multicolumn{6}{|l|}{ HIV subtype } \\
\hline $\mathrm{B}$ & $194(64.9 \%)$ & $32(29.9 \%)$ & $92(83.6 \%)$ & $70(85.4 \%)$ & 0.013 \\
\hline CRF07_BC & $101(33.8 \%)$ & $74(69.2 \%)$ & $17(15.5 \%)$ & $10(12.2 \%)$ & $<0.001$ \\
\hline CRF01_AE & $3(1.0 \%)$ & $1(0.9 \%)$ & $1(0.9 \%)$ & $1(1.2 \%)$ & 0.743 \\
\hline Others & $2(0.7 \%)$ & $1(0.9 \%)$ & $0(0.0 \%)$ & $1(1.2 \%)$ & \\
\hline
\end{tabular}

${ }^{a} \mathrm{CRF}$, circulating recombinant form; MSM, men having sex with men; IDU, intravenous drug user; MTCT, mother-to-child transmission. $\mathrm{b}_{p}$ value indicates a significantly difference $(p<0.05)$ between 2007 and 2009. 
In summary, our Harm Reduction program has been shown to decrease the spread of HIV among IDUs. Nevertheless, the proportion of recent infections in IDUs increased from 2008 to 2009 . Therefore, to carry out an NSP thoroughly and continuously was important to decrease the further spread of HIV in IDUs. In addition, continuous ongoing monitoring of the molecular epidemiology of HIV can be useful to monitor the spread of HIV in order to establish and modify public health efforts to prevent new infections.

\section{Acknowledgments}

We thank Ms. Ying-Chen Lin of the HIV / AIDS Control and Study Center of National Taiwan University and Ms. KuanLin Chen of the Taiwan Centers of Disease Control for their technical assistance. This research was supported by funds from the Taiwan Centers for Disease Control (DOH97-DC2011, 98-0324-01-F-20 and DOH98-DC-2016). The first three authors contributed equally to this work.

\section{Author Disclosure Statement}

No competing financial interests exist.

\section{References}

1. Weniger BG, Takebe Y, Ou CY, et al.: The molecular epidemiology of HIV in Asia. AIDS 1994;8(Suppl 2):S13-28.

2. Chuang CY, Chang PY, and Lin KC: AIDS in the Republic of China, 1992. Clin Infect Dis 1993;17(Suppl 2):S337-340.

3. Taiwan Centers for Disease Control: HIV/AIDS data, 2010: http://www.cdc.gov.tw/public/Attachment/0461675471.xls.

4. Lan YC, Elbeik T, Dileanis J, et al.: Molecular epidemiology of HIV-1 subtypes and drug resistant strains in Taiwan. J Med Virol 2008;80(2):183-191.

5. Chen YM and Kuo SH: HIV-1 in Taiwan. Lancet 2007;369 (9562):623-625.

6. Chang SY, Sheng WH, Lee CN, et al:: Molecular epidemiology of HIV type 1 subtypes in Taiwan: Outbreak of HIV type 1 CRF07_BC infection in intravenous drug users. AIDS Res Hum Retroviruses 2006;22(11):1055-1066.

7. Chen YM, Lan YC, Lai SF, et al.: HIV-1 CRF07_BC infections, injecting drug users, Taiwan. Emerg Infect Dis 2006;12(4): 703-705.

8. Lin HH, Shih YL, Liu YC, et al.: An epidemic of HIV type I CRF07_BC infection among injection drug users in Taiwan. J Acquir Immune Defic Syndr. 2006;42(2):248-255.

9. Central Asia: Several HIV outbreaks linked to transfusions of tainted blood and poor sanitary conditions in hospitals. HIV AIDS Policy Law Rev 2007;12(2-3):57-58.
10. Yang $\mathrm{CH}$, Yang $\mathrm{SY}$, Shen $\mathrm{MH}$, et al.: The changing epidemiology of prevalent diagnosed HIV infections in Taiwan, 1984-2005. Int J Drug Policy 2008;19(4):317-323.

11. Parekh BS, Kennedy MS, Dobbs T, et al.: Quantitative detection of increasing HIV type 1 antibodies after seroconversion: A simple assay for detecting recent HIV infection and estimating incidence. AIDS Res Hum Retroviruses 2002;18(4):295-307.

12. Dobbs T, Kennedy S, Pau CP, et al.: Performance characteristics of the immunoglobulin G-capture BED-enzyme immunoassay, an assay to detect recent human immunodeficiency virus type 1 seroconversion. J Clin Microbiol 2004; 42(6):2623-2628.

13. Thompson JD, Higgins DG, and Gibson TJ: CLUSTAL W: Improving the sensitivity of progressive multiple sequence alignment through sequence weighting, position-specific gap penalties and weight matrix choice. Nucleic Acids Res 1994;22(22):4673-4680.

14. Tamura K, Dudley J, Nei M, and Kumar S: MEGA4: Molecular Evolutionary Genetics Analysis (MEGA) software version 4.0. Mol Biol Evol 2007;24(8):1596-1599.

15. Felsenstein J: PHYLIP-Phylogeny Inference Package (Version 3.2). Cladistics 1989;5:164-166.

16. Taiwan Centers for Disease Control: HIV/AIDS data, 2010: http://www.cdc.gov.tw/public/Attachment/01612104271 .xls.

17. Taiwan Centers for Disease Control: Taiwan Government's Responses to HIV/AIDS, 2007: http://www.cdc.gov.tw/ content.asp?CuItem $=7424$.

18. Chen YM, Huang KL, Jen I, et al.: Temporal trends and molecular epidemiology of HIV-1 infection in Taiwan from 1988 to 1998. J Acquir Immune Defic Syndr 2001;26(3):274-282.

19. Lin YT, Lan YC, Chen YJ, et al:: Molecular epidemiology of HIV-1 infection and full-length genomic analysis of circulating recombinant form 07_BC strains from injection drug users in Taiwan. J Infect Dis 2007;195(9):1283-1293.

20. Chen YJ, Huang YH, Chuang SY, et al:: Molecular epidemiology of HIV-1 subtype B, CRF01_AE, and CRF07_BC infection among injection drug users in Taiwan. J Acquir Immune Defic Syndr 2010;53(4):425-439.

Address correspondence to: Jyh-Yuan Yang

Centers for Disease Control Taiwan R.O.C., No. 161 Kun-Yang Street

Taipei

Taiwan

E-mail: jyyang@cdc.gov.tw 
